\title{
MODELOS ARQUITETÔNICOS PARA AS ESPÉCIES ARBÓREAS DE UMA FLORESTA ESTACIONAL SUBTROPICAL NO RIO GRANDE DO SUL
}

\author{
ARCHITECTURAL MODELS OF THE TREE SPECIES OF THE SUBTROPICAL SEASONAL \\ FOREST IN THE STATE OF RIO GRANDE DO SUL, BRAZIL
}

\author{
Malcon do Prado Costa ${ }^{1}$ Solon Jonas Longhi ${ }^{2}$
}

\begin{abstract}
RESUMO
Os modelos arquitetônicos ajudam a descrever o desenvolvimento e a organização das copas das árvores. Este conhecimento é essencial para a compreensão do desenvolvimento das árvores na floresta e pode ajudar a entender a relação evolutiva entre táxons. $O$ trabalho teve como objetivo verificar a riqueza de modelos arquitetônicos das espécies arbóreas de uma Floresta Estacional. Os dados foram coletados a partir de observações de campo e literatura especializada. Foram encontradas 53 espécies da flora arbórea de uma Floresta Estacional Subtropical de Santa Maria - RS, distribuídas em 45 gêneros e 28 das famílias. No total, 13 diferentes modelos arquitetônicos foram reconhecidos. Os modelos com maior destaque foram Rauh, Troll, Scarrone, Roux e Massart. A complexidade arquitetural, refletida pelo elevado número de modelos descritos, se deve ao desenvolvimento do dossel no remanescente estudado. O trabalho teve um caráter pioneiro e exploratório e deve fornecer um marco para novos estudos sobre arquitetura de plantas na região subtropical do Brasil.
\end{abstract}

Palavras-chave: arquitetura arbórea; silvologia; chaves dicotômicas.

\begin{abstract}
Architectural models contribute to describe the development and the arrangement of the tree canopy. This knowledge is crucial for the understanding of the behavior of forest trees and it may also help us understand the evolutionary relationships among the taxa. This study is aimed at identifying how rich the architectural models of the arboreal species of a seasonal forest are. The data was gathered from field observations and specialized literature. It presents a list of 53 tree species from the tree flora of the Subtropical Seasonal Forest of the Santa Maria - RS, distributed in 45 genera and 28 families in the tree flora. A total of 13 different architectural models have been recognized. The most prominent models were Rauh, Troll, Scarrone, Roux and Massart. The architectural complexity, reflected by the high number of models described, is due to the development of the canopy in the rest of the study. This work had a pioneering and exploratory character and shall provide a framework for new studies on plant architecture in the subtropical region of Brazil.
\end{abstract}

Keywords: tree architecture; silvology; dichotomous keys.

\section{INTRODUÇÃO}

A análise arquitetural das árvores ajuda a descobrir e examinar parte da história e qualidade de vida da planta (HALLÉ; OLDEMAN; TOMLINSON, 1978), é uma ferramenta para encontrar e estabelecer relações evolutivas (VESTER, 1999), possui utilidade em estudos taxonômicos (TOURN; BARTHÉLÉMY; GROSFELD, 1999) e ajuda a interpretar a dinâmica da comunidade florestal a partir do reconhecimento dos padrões de desenvolvimento da arquitetura das árvores individuais (TORQUEBIAU, 1986; VESTER,

1 Engenheiro Florestal, Dr., Professor do Programa de Pós-Graduação em Ciências Florestais, Escola Agrícola de Jundiaí, Unidade Acadêmica Especializada em Ciências Agrárias, Universidade Federal do Rio Grande do Norte, RN 160 - Km 03, Jundiaí, CEP 59280-000, Macaíba (RN), Brasil. malconfloresta@gmail.com

2 Engenheiro Florestal, Dr., Professor Titular do Departamento de Ciências Florestais, Centro de Ciências Rurais, Universidade Federal de Santa Maria, Av. Roraima, 1000, CEP 97105-900, Santa Maria (RS), Brasil. longhi. solon@gmail.com

Recebido para publicação em 15/04/2014 e aceito em 8/02/2018

Ci. Fl., v. 28, n. 4, out. - dez., 2018 
1997; VESTER; CLEEF, 1998). Durante a evolução, as formas das árvores se modificam interagindo com a estrutura da floresta (OLDEMAN, 1990; VESTER, 1999).

O estudo de arquitetura de plantas, como um método para a análise e compreensão do desenvolvimento biológico das árvores, foi consolidado como uma disciplina a partir da década de 70, com o livro sobre modelos arquitetônicos de Hallé, Oldeman e Tomlinson (1978). Os autores proporcionaram a estrutura teórica para o estudo do crescimento e desenvolvimento de plantas, em termos de unidades biologicamente significativas e visualmente diferenciáveis, e como resultado, foi gerado um novo sentido para a noção de indivíduo e população de plantas (TORQUEBIAU, 1979; BARTHÉLÉMY; CARAGLIO, 2007).

Um elemento-chave no estudo da arquitetura de plantas é o reconhecimento inicial de um padrão de ramificação primordial, que se expressa entre a germinação e a floração, e pode ser descrito como um modelo arquitetônico (HALLÉ; OLDEMAN; TOMLINSON, 1978) ou unidade arquitetônica (PERRETA; VEGETTI, 2005). Com base no modelo arquitetônico pode-se examinar qualquer árvore, em sua história de vida, e confeccionar um diagrama com o padrão de desenvolvimento de uma espécie, da plântula até sua senescência. Se usam conceitos como o de reiteração (HALLÉ; OLDEMAN; TOMLINSON, 1978), significando a repetição de um modelo dentro do mesmo modelo, total ou parcialmente, de acordo com pressões ambientais, e o de metamorfose, processo de reiteração programada de acordo com o desenvolvimento da árvore (OLDEMAN, 1990; TOURN; BARTHÉLÉMY; GROSFELD, 1999).

A arquitetura de uma planta, em um dado momento, é a expressão de um equilíbrio entre os processos de crescimento endógeno e as forças externas exercidas pelo ambiente (BARTHÉLÉMY; CARAGLIO, 2007). O objetivo da análise arquitetônica é identificar os processos endógenos que determinam a forma de uma árvore.

Para entender a arquitetura de uma planta analisa-se a atividade dos meristemas, principalmente os apicais. A base para a análise das árvores são os eixos de crescimento, produto da atividade de apenas um meristema apical caulinar, com suas respectivas características quanto à orientação e posição dos órgãos, como as folhas e as flores. A caracterização do eixo principal de uma planta e suas ramificações de primeira ordem forma uma combinação característica para uma espécie, a qual se conhece como modelo arquitetônico. Hallé, Oldeman e Tomlinson (1978) citam a possibilidade de 24 modelos teóricos possíveis, dos quais apenas 23 foram reconhecidos.

A existência de modelos arquitetônicos intermediários se deve, em parte, à capacidade de muitas árvores repetir o desenvolvimento de suas estruturas, cada uma repetindo o modelo básico da árvore dentro da mesma árvore - processo denominado como reiteração. O resultado da reiteração é uma copa muito maior e completa do que um modelo básico pode produzir por si próprio, e assegura a substituição da copa quando danificada. A reiteração se expressa de diferentes formas, uma delas modificando as características dos eixos secundários e ao longo do eixo principal. Esta forma de reiteração é denominada metamorfose. Durante este processo, a árvore modifica seu modelo arquitetônico. Uma forma de expressar esta mudança é considerar o modelo como intermediário, outra, é descrever a arquitetura com mais detalhes durante sua história de vida (HALLÉ; OLDEMAN; TOMLINSON, 1978; OLDEMAN, 1990; TOURN; BARTHÉLÉMY; GROSFELD, 1999).

Os modelos arquitetônicos não são categorias ou classes, mas sim áreas de sobreposição em um contínuo da construção arquitetural da planta. Desta maneira, os descritores dos modelos, como por exemplo, a plagiotropia e a ortotropia são extremos de um contínuo de diferenciação. Junto com a reiteração, a continuidade entre os descritores é uma causa da existência de modelos intermediários (HALLÉ; OLDEMAN; TOMLINSON, 1978).

Parte do interesse em modelos arquitetônicos é o seu relacionamento com a ecologia (TORQUEBIAU, 1986; OLDEMAN, 1990; VESTER; SALDARRIAGA, 1993; VESTER, 1997; VESTER; CLEEF, 1998). Globalmente foi observado que a maior concentração de modelos ocorre nos trópicos. De acordo com Hallé, Oldeman e Tomlinson (1978), a quantidade de modelos arquitetônicos diminui com o aumento das intempéries e fatores restritivos do ambiente, por isso esperam-se encontrar menor riqueza de modelos em florestas estacionais, devido à pronunciada estação seca, em comparação com floresta pluviais. É o caso da floresta estacional estudada, que está inserida em clima subtropical, caracterizado por apresentar uma estação de frio intenso, o qual ocasiona seca fisiológica, provocando a perda das folhas em algumas espécies vegetais (LEITE; KLEIN, 1990). 
Esta pesquisa possui um caráter pioneiro devido à carência de estudos sobre arquitetura de plantas no Brasil, de referências bibliográficas em literatura de língua portuguesa, e por realizar a primeira classificação e listagem de modelos de arquitetura arbórea de uma Floresta Estacional Subtropical, na cidade de Santa Maria - RS. O trabalho teve o objetivo de verificar a riqueza de modelos arquitetônicos das espécies arbóreas de uma Floresta Estacional e comparar a riqueza de modelos arquitetônicos desta tipologia florestal com aqueles registrados em uma Floresta Estacional na Península Yucatán, no México e em uma Floresta Pluvial, na Amazônia Colombiana.

\section{METODOLOGIA}

Os dados foram coletados em remanescentes da Floresta Estacional Subtropical, no município de Santa Maria, no período 2011-2013. Foram observados 30 indivíduos da mesma espécie em diferentes fases de desenvolvimento da planta, com o objetivo de acumular a informação necessária para determinar o modelo arquitetônico. Também foram examinadas classificações de modelos arquiteturais encontrados em literatura (HALLÉ; OLDEMAN; TOMLINSON, 1978; VESTER; SALDARRIAGA, 1993; VESTER, 1997). As espécies foram observadas em um remanescente de Floresta Estacional Subtropical, no Campo de Instrução de Santa Maria do Exército Brasileiro (CISM). As observações ocorreram diretamente em árvores pequenas (Altura Total $<5 \mathrm{~m}$ ) e por meio de binóculos para árvores grandes (Altura total $>5 \mathrm{~m}$ ). Nas espécies com identidade desconhecida, foram coletadas amostras de ramos para sua determinação, que foram depositadas no Herbário do Departamento de Ciências Florestais (HCDF) da UFSM.

A definição de árvore, utilizada no presente trabalho, está referenciada nas obras de Hallé, Oldeman e Tomlinson (1978) e Oldeman (1990), que a definem como todo organismo vegetal que vive seu ciclo de vida de acordo com uma estratégia, incluindo a maximização do seu tempo e vida, investindo matéria e energia na formação de tecidos de autossustentação e de transporte em quantidades importantes em relação à sua biomassa total, sobressaindo-se à vegetação circundante a partir de um programa adaptado de crescimento em camadas.

Foram utilizadas as seguintes características para distinguir os diferentes tipos de eixo de uma planta (Figura 1):

Diferenciação: a ortotropia - resposta à gravidade em que o eixo vertical produz os seus ramos - se reconhece pelo desenvolvimento vertical e a orientação das folhas em espiral ou oposta (eixo ortotrópico - Figura 1A), e a plagiotropia - resposta à gravidade que os eixos horizontais ou oblíquos produzem seus ramos se reconhece com simetria dorsiventral e em forma dística no extremo (eixo plagiotrópico - Figura 1B). Existem muitas formas intermediárias entre estes extremos. Por isto é sugerido que o eixo plagiotrópico é mais diferenciado do que o ortotrópico (HALLÉ; OLDEMAN; TOMLINSON, 1978). Neste trabalho considerou-se como eixo plagiotrópico qualquer eixo que tenha algum indício de plagiotropia, seja por sua orientação obliqua ou pela orientação das folhas em um plano.

Tipo de ramificação: presente ou ausente, rítmica ou contínua. Muitas palmeiras e outras monocotiledôneas não apresentam nenhuma forma evidente de ramificação. Elas se formam pela atividade de um único meristema que não se ramifica. Outras plantas podem ter tanto eixos que se ramificam como outros que não o fazem. A ramificação pode ocorrer em todas as axilas das folhas que formam o meristema (ramificação contínua - Figura 1C), ou pode ocorrer em zonas definidas e repetidas ao longo do eixo (ramificação rítmica - Figura 1D).

Tipo de crescimento: contínuo ou rítmico. Se refere à atividade recorrente de crescimento e descanso do meristema apical, geralmente visível pela distribuição de folhas pequenas e grandes ao longo do eixo. O ritmo de crescimento é diferente do ritmo de ramificação, sendo que a maioria das espécies que apresenta crescimento contínuo, apresenta também ramificação contínua, com algumas exceções em que espécies com crescimento contínuo apresentam ramificação rítmica. Para a identificação do modelo arquitetônico, o ritmo da ramificação tem prioridade, pois expressa uma atividade rítmica do meristema. 
Crescimento determinado ou indeterminado: o primeiro se refere à conversão do meristema apical em inflorescência (E), gavinha ou bola de parênquima $(F)$, ou outra estrutura que não permite que o eixo siga se alongando. Os eixos com crescimento indeterminado não possuem um fim determinado em seu funcionamento. Um tronco ou um ramo formado pelo encadeamento de eixos com crescimento determinado se conhece como ramo ou tronco simpodial enquanto um tronco ou ramo formado por um só eixo indeterminado é um ramo ou tronco monopodial. Um tronco simpodial também é formado pelo encadeamento de eixos mistos (HALLÉ, 2009).

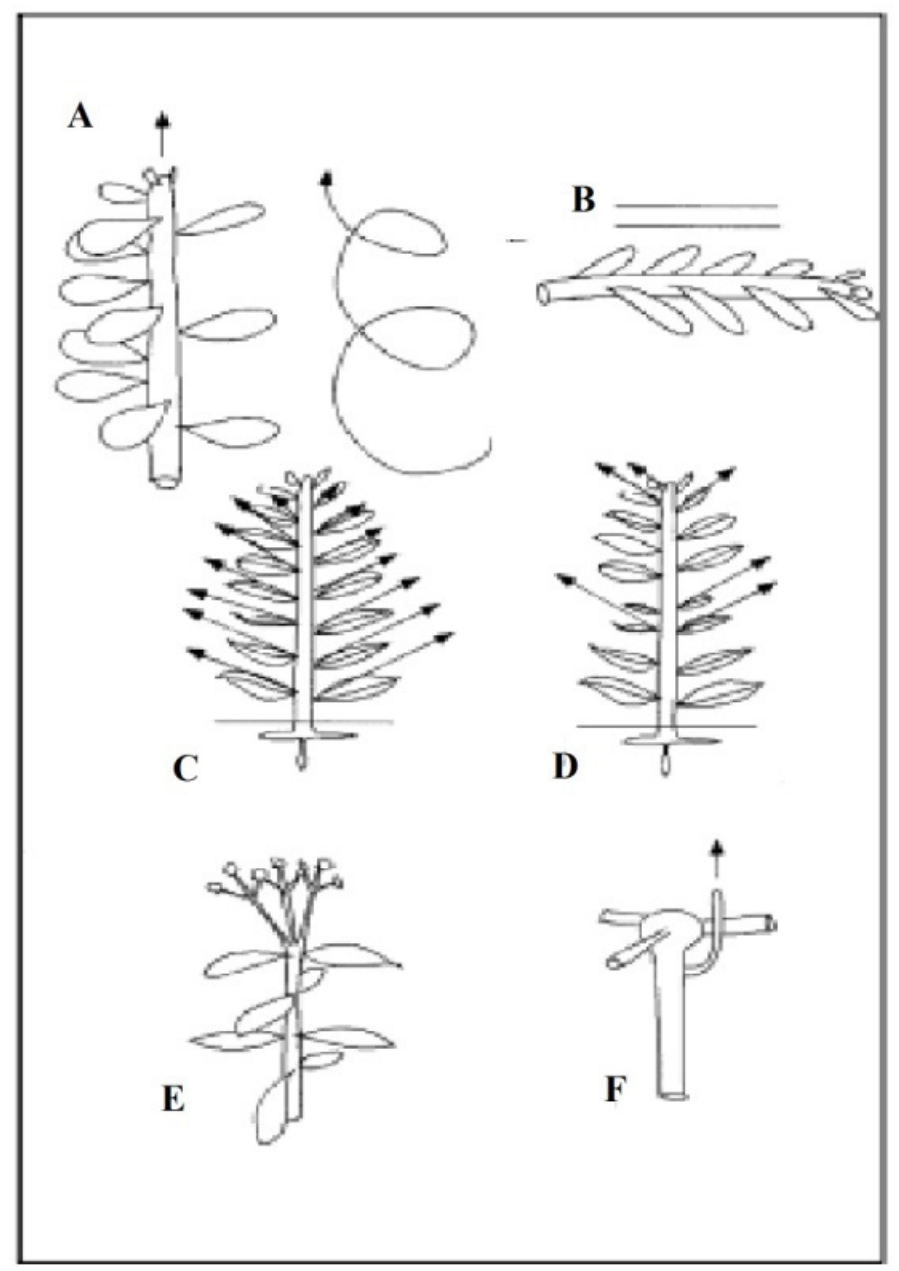

FIGURA 1: Caracteres dos eixos usados nas análises arquitetônicas (VESTER, 2002). A) O eixo ortotrópico tem um desenvolvimento vertical e a orientação das folhas em espiral; B) O eixo plagiotrópico tem uma orientação horizontal e as folhas em um mesmo plano, em filas; C) Ramificação contínua; D) Ramificação rítmica; E) Crescimento determinado, com o eixo terminando em uma inflorescência; F) Crescimento determinado, como no modelo de Prevost (Cordia americana), terminando em uma bola de parênquima.

FIGURE 1: Characters of axes used in architectural analysis (VESTER, 2002). A) The orthotropic axis has a vertical development and a leaf orientation by spiral phyllotaxy; B) The plagiotrophycal axis has a horizontal orientation and leaves on the same plane; C) Continuous branching; D) Rhythmic branching; E) Growth determined, with the axis ending in an inflorescence; F) Determinate growth, like a Prevost model (Cordia americana), ending in a parenchyma.

Para determinar o modelo arquitetônico foram realizadas as seguintes observações: tipo de crescimento: rítmico ou contínuo; tipo de ramificação: rítmica ou contínua; caracterização dos eixos principais: ortotrópicos, plagiotrópicos ou mistos; caracterização dos eixos secundários: ortotrópicos, 
plagiotrópicos ou mistos; posição das flores ou inflorescências: terminais ou laterais. As definições de Hallé, Oldeman e Tomlinson (1978) foram usadas para a identificação dos modelos arquitetônicos (Tabela 1). Nas seguintes caracterizações foi usado o conceito de eixo, de acordo com a abordagem descrita acima, como o produto de um único meristema apical. $O$ termo tronco refere-se ao eixo ou encadeamento do eixo principal no modelo. O termo ramo se refere à estrutura secundária axilar no modelo. Na maioria de vezes, uma árvore se constrói pela multiplicação de réplicas do modelo, denominadas reiterações.

TABELA 1: Critérios e características que definem os modelos arquitetônicos. Modelos arquitetônicos, segundo Hallé, Oldeman e Tomlinson (1978). A) Modelos sem ramificação; B) Modelos com ramificação sem diferenciação entre os eixos; C) Modelos com diferenciação entre os eixos; D) Modelos com eixos mistos, formando tronco e ramo (Ilustração adaptada - OLDEMAN, 1990; VESTER, 2002).

TABLE 1: Criteria and traits that define architectural models. Architectural models agreed Hallé, Oldeman e Tomlinson (1978). A) Models without branching; B) Models with branching and without differentiating between the axes; C) Models with differentiating between the axes; D) Models with mixed axes, forming stem and branch (Illustration adapted - OLDEMAN, 1990; VESTER, 2002).

\section{A - Modelos sem ramificação}

Holttum: Consta de um único meristema que forma um eixo não ramificado, com crescimento determinado por uma inflorescência terminal.

Corner: É caracterizado por um crescimento vegetativo com meristemаноцтuм que produz um eixo não ramificado, com crescimento indeterminado, no qual a inflorescência é lateral.

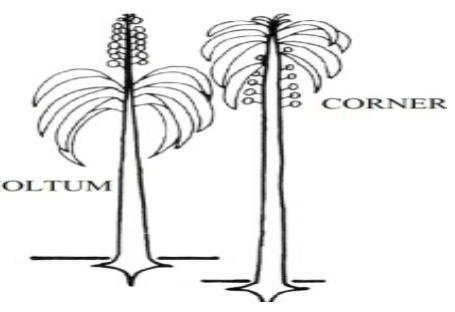

\section{B - Modelos ramificados sem diferenciação entre os eixos}

Chamberlain: Superposição determinada dos eixos de crescimento, formando um tronco simpodial ascendente linear.

Schoute: Crescimento por meio de meristemas que formam eixos ortotrópicos ou plagiotrópicos por dicotomia, ou seja, a divisão do meristema apical em dois novos meristemas equivalentes. As inflorescências são laterais.

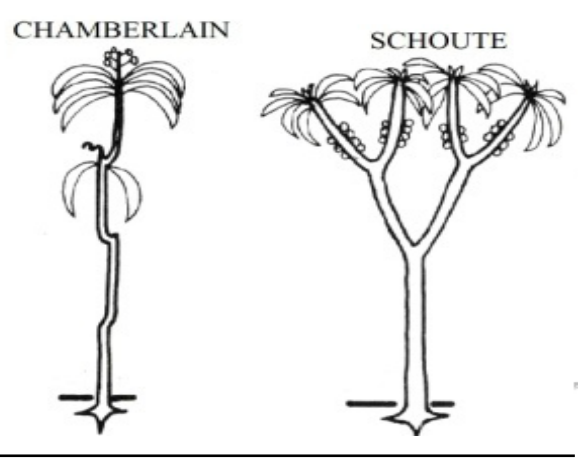

Leeuwenberg: Consta de eixos ortotrópicos equivalentes com crescimento determinado pela produção de uma inflorescência terminal. A ramificação produz vários eixos equivalentes que, por sua vez, são ortotrópicos e de crescimento determinado. A ramificação está relacionada com a inflorescência, ocorrendo após o florescimento, e em posição periférica, debaixo da inflorescência, exceto nos eixos juvenis, nos quais pode haver ramificação sem inflorescência. Nestes casos, o meristema terminal geralmente deixa de funcionar.

Tomlinson: Arquitetura que resulta do desenvolvimento repetitivo dos eixos ortotrópicos equivalentes, de ramificação basal. As inflorescências podem ser terminais ou laterais; o crescimento de cada eixo pode ser

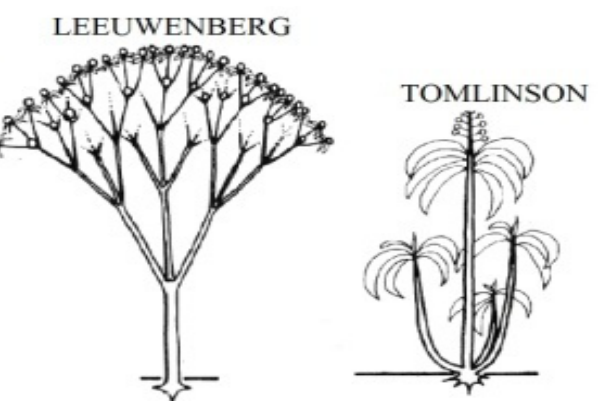
contínuo ou rítmico. 
TABELA 1: Continuação...

TABLE 1: Continued...

\section{C - Modelos com diferenciação entre eixos}

McClure: Possui dois tipos de eixos. Os troncos são ortotrópicos e se originam por ramificação basal; apicalmente apresentam ramos plagiotrópicos. Ambos os tipos de eixos possuem crescimento determinado e um alto grau de pré-formação. Ou seja, significa que na gema já estão formadas todas as partes dos eixos antes do crescimento em extensão. Ecologicamente significa que, durante a extensão, não pode haver ajustes maiores na organização do eixo.

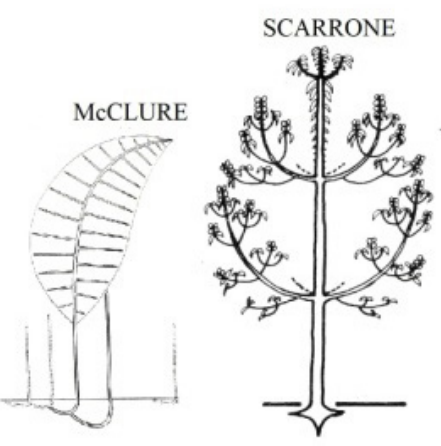

Scarrone: Arquitetura caracterizada por um tronco ortotrópico com crescimento rítmico, formando pseudoverticilos de ramos ortotrópicos e simpodiais por substituição, com inflorescências terminais.

Stone: Forma-se por um tronco ortotrópico com crescimento contínuo e ramos ortotrópicos, simpodiais por substituição. As inflorescências são terminais.

Attim: Forma-se por um eixo principal ortotrópico, com crescimento e ramificação contínua, sendo os ramos também ortotrópicos. As inflorescências são laterais.

Rauh: Este modelo possui a arquitetura determinada por um tronco monopodial com crescimento rítmico, em que se desenvolvem pseudoverticilos de ramos morfogeneticamente iguais ao tronco. A posição das flores ou inflorescências são, sempre, laterais.

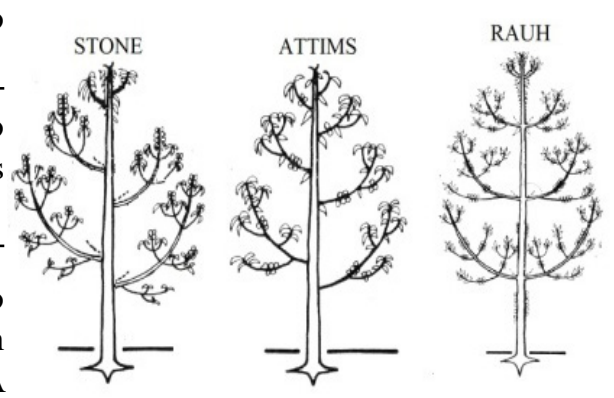

Koriba: Este modelo se caracteriza por eixos ortotrópicos com diferenciação secundária entre eles. O primeiro eixo é ortotrópico com ramificação distal, produzindo uma série de ramos que a princípio são equivalentes, e também ortotrópicos. Um destes ramos se torna dominante, funcionando como o eixo principal, os outros funcionam como ramos. As inflorescências são terminais.
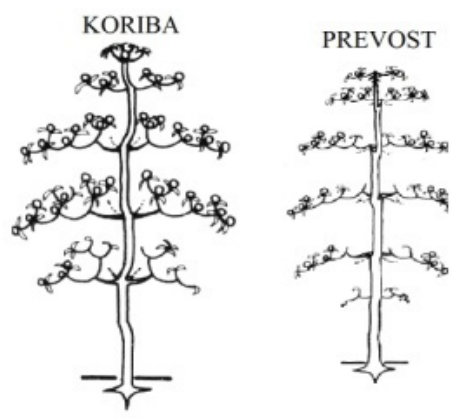

Prevost: Modelo que forma um tronco simpodial, com eixos crescendo de forma determinada. Geralmente, os eixos terminam em uma bola de parênquima. Os ramos se originam em uma área subapical (siléptico) restringida do tronco e são simpodiais com eixos mistos plágioortotrópicos com crescimento determinado, geralmente terminando na inflorescência. Este modo de formação de ramos plagiotrópicos por eixos mistos de crescimento determinado é denominado plagiotropia por substituição. O tronco se forma pela superposição de eixos que nascem debaixo dos verticilos do ramo (proléptico). 
TABELA 1: Continuação...

TABLE 1: Continued...

Fagerlind: Arquitetura caracterizada por um tronco monopodial (formado por um só eixo), ortotrópico, com crescimento rítmico e verticilos ou pseudoverticilos de ramos plagiotropicossimpodiais. Cada ramo se forma a partir de plagiotropia por substituição.
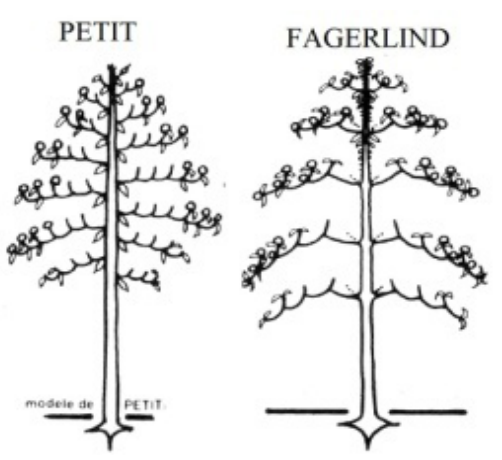

Petit: Arquitetura caracterizada por um crescimento contínuo de um tronco monopodial, ortotrópico, que produz continuamente ramos plagiotropicossimpodiais com folhas em espiral, ou opostas com crescimento determinado, terminando na inflorescência (ramo simpodialplagiotrópico por substituição).

Aubreville: Arquitetura caracterizada por um tronco monopodial ortotrópico com crescimento rítmico e filotaxia em espiral ou oposta. Este tronco possui pseudoverticilos de ramos simpodiais, cada um constituído por eixos mistos plágio-ortótropos, com floração lateral. Este modo de formação de ramo plagiotrópico por eixos de crescimento indeterminado se denomina plagiotropia por justaposição.

Nozeran: A arquitetura é caracterizada por um tronco ortotrópico, simpodial, em que cada eixo simpodial apresenta crescimento determinado, geralmente em uma bola de parênquima e em posição apical um verticilo de ramos plagiotrópicos; a plagiotropia está estabelecida pela posição das folhas e não por superposição ou substituição. Os eixos podem ter ou não crescimento rítmico. Os ramos podem ser simpodiais ou monopodiais e a posição da inflorescência não influencia o modelo.

Roux: Este modelo possui uma arquitetura determinada por um tronco ortotrópico monopodial com crescimento contínuo; os ramos são plagiotrópicos e se inserem no tronco de forma contínua. A localização da inflorescência não é relevante para este modelo.

Cook: É igual ao modelo de Roux, pois os ramos são filiformes, ou seja, parecem folhas compostas; possuem um crescimento determinado sem formar ramos simpodiais.

Massart: Este modelo apresenta arquitetura caracterizada por um tronco monopodial ortotrópico, com crescimento rítmico e filotaxia espiral ou
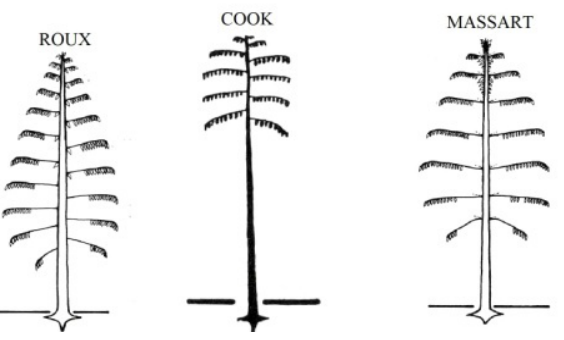
oposta. Este tronco carrega pseudoverticilos de ramos plagiotrópicos. A
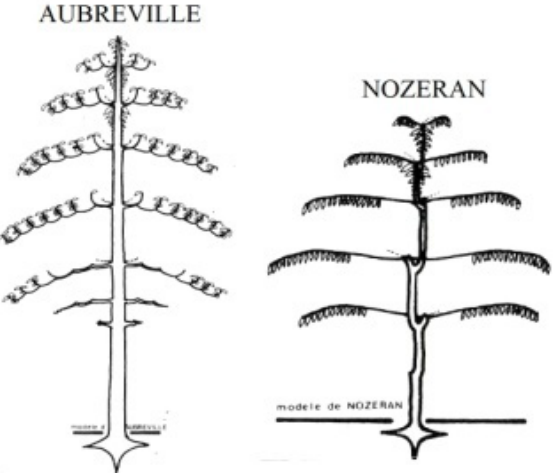
posição da inflorescência não influencia o modelo. 
TABELA 1: Continuação...

TABLE 1: Continued...

\section{D - Modelos com eixos mistos}

Mangenot: Define-se por eixos mistos. O meristema apical produz primeiro uma parte basal vertical, logo uma parte periférica horizontal, muitas vezes relacionada com mudanças em sua filotaxia, de espiral a dística, e de folhas pequenas a grandes. A superposição indeterminada de tais eixos, em nível da transição de vertical à horizontal, constrói a arquitetura da árvore, com o tronco formado por o encadeamento sucessivo das partes verticais próximas de cada um dos eixos. Os ramos resultam das partes periféricas horizontais de cada eixo.
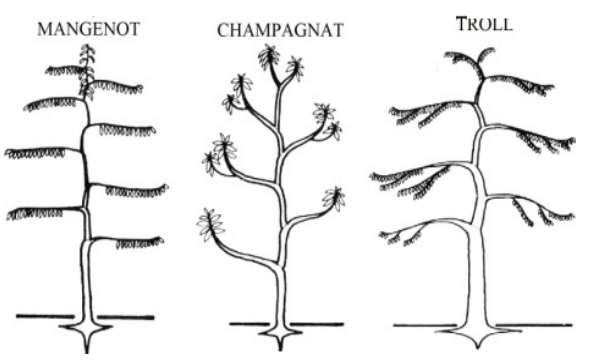

Champagnat: Define-se pela superposição dos eixos mistos ortotrópicos. As partes periféricas tornam-se pêndulas e formam os ramos. As partes basais formam um tronco simpodial.

Troll: Este modelo apresenta eixos mistos. Define-se por eixos plagiotrópicos. A arquitetura está construída pela sobreposição contínua destes eixos. Os principais eixos constituem parte do tronco e do ramo; a parte próxima ao eixo cresce ereta (parte do tronco). A parte distante forma um ramo com ou sem crescimento determinado, e com os eixos laterais em geral sem formar uma parte basal ereta.

A partir da classificação das espécies arbóreas de acordo com seus modelos arquitetônicos foram elaboradas chaves dicotômicas, com o uso de caracteres morfológicos e arquiteturais das plantas inventariadas.

\section{RESULTADOS E DISCUSSÃO}

As 52 espécies (44 gêneros de 28 famílias) avaliadas neste estudo foram enquadradas em 13 modelos arquitetônicos (Tabela 2). O modelo de Rauh foi o que envolveu um maior número de espécies, entre elas: Actinostemon concolor, Aiouea saligna, Erythroxylum deciduum, Eugenia uniflora, Ficus luschnathiana, Helietta apiculata, Maclura tinctoria, Sebastiania brasiliensis, Gymnanthes klotzschiana, Trichilia catigua, Trichilia clausseni, Trichilia elegans, Vitex megapotamica.

Nos modelos sem diferenciação entre os eixos, as espécies Pisonia ambigua e Schefflera calva foram classificadas no modelo de Leeuwenberg. Entre os modelos com diferenciação entre os eixos, foram classificadas as espécies Handroanthus heptaphyllus e Phytolacca dioica no modelo de Koriba. As espécies do gênero Cordia, no modelo de Prevost. Citronela paniculata, no modelo de Nozeran. A espécie Diospyros inconstans, assim como as Mirtáceas Eugenia involucrata, Eugenia ramboi e Myrcianthes pungens foram classificadas no modelo de Massart. 
TABELA 2: Padrão de ramificação (P.R.) dos eixos, modelos arquitetônicos (M.A.), famílias botânicas, espécies arbóreas e seus respectivos nomes vernaculares.

TABLE 2: Branching pattern (P.R.), architectural model (M.A.), botanical families, tree species and their vernacular names.

\begin{tabular}{|c|c|c|c|c|}
\hline P.R. & M.A. & Família & Nome científico & Nome vernacular \\
\hline A & Corner & ARECACEAE & Syagrus romanzoffiana (Cham.) Glassman & Jerivá \\
\hline B & Leeuwenberg & ARALIACEAE & Schefflera calva (Cham.) Fordin \& Fiaschi & Caixeta \\
\hline B & Leeuwenberg & NYCTAGINACEAE & Pisonia ambigua Heirmerl & Anzol-de-lontra \\
\hline $\mathrm{C}$ & Attim & ASTERACEAE & Moquiniastrum polymorphum (Less.) G.Sancho & Cambará \\
\hline $\mathrm{C}$ & Aubreville & SOLANACEAE & Solanum sanctaecatharinae Dunal & Canena \\
\hline $\mathrm{C}$ & Aubreville & URTICACEAE & Boehmeria caudata $\mathrm{Sw}$. & Urtiga-mansa \\
\hline $\mathrm{C}$ & Aubreville & URTICACEAE & Urera baccifera (L.) Gaudich. ex Wedd. & Urtigão-bravo \\
\hline $\mathrm{C}$ & Koriba & BIGNONIACEAE & Handroanthus heptaphyllus (Vell.) Mattos & Ipê-roxo \\
\hline $\mathrm{C}$ & Koriba & PHYTOLACCACEAE & Phytolacca dioica $\mathrm{L}$ & Umbú \\
\hline $\mathrm{C}$ & Massart & EBENACEAE & Diospyros inconstans Jacq. & Maria-preta \\
\hline $\mathrm{C}$ & Massart & MYRTACEAE & Eugenia involucrata DC. & $\begin{array}{l}\text { Cerejeira-do- } \\
\text { mato }\end{array}$ \\
\hline $\mathrm{C}$ & Massart & MYRTACEAE & Eugenia ramboi D.Legrand & Batinga-branca \\
\hline $\mathrm{C}$ & Massart & MYRTACEAE & Myrcianthes pungens (O. Berg) D.Legrand & Guabijú \\
\hline $\mathrm{C}$ & Nozeran & CARDIOPTERIDACEAE & Citronella paniculata (Mart.) R.A.Howard & Pau-de-corvo \\
\hline $\mathrm{C}$ & Prevost & BORAGINACEAE & Cordia americana (L.) Gottschling \& J.S.Mill. & Guajuvira \\
\hline $\mathrm{C}$ & Prevost & BORAGINACEAE & Cordia trichotoma (Vell.) Arráb. ex Steud. & Louro-pardo \\
\hline $\mathrm{C}$ & Rauh & ERYTHROXYLACEAE & Erythroxylum deciduum A.St.-Hil. & Cocão \\
\hline $\mathrm{C}$ & Rauh & EUPHORBIACEAE & Actinostemon concolor (Spreng.) Müll.Arg. & $\begin{array}{l}\text { Laranjeira-do- } \\
\text { mato }\end{array}$ \\
\hline $\mathrm{C}$ & Rauh & EUPHORBIACEAE & Sebastiania brasiliensis Spreng. & $\begin{array}{l}\text { Branquilho- } \\
\text { leiteiro }\end{array}$ \\
\hline $\mathrm{C}$ & Rauh & EUPHORBIACEAE & Gymnanthes klotzschiana Müll.Arg. & Branquilho \\
\hline $\mathrm{C}$ & Rauh & LAMIACEAE & Vitex megapotamica (Spreng.) Moldenke & Tarumã \\
\hline $\mathrm{C}$ & Rauh & LAURACEAE & Aiouea saligna Meisn. & Canela-vermelha \\
\hline $\mathrm{C}$ & Rauh & MELIACEAE & Trichilia catigua A.Juss. & Catiguá \\
\hline $\mathrm{C}$ & Rauh & MELIACEAE & Trichilia clausseni C.DC. & Catiguá-vermelho \\
\hline $\mathrm{C}$ & Rauh & MELIACEAE & Trichilia elegans A.Juss. & Pau-de-ervilha \\
\hline $\mathrm{C}$ & Rauh & MORACEAE & Ficus luschnathiana (Miq.) Miq. & Gameleira \\
\hline $\mathrm{C}$ & Rauh & MORACEAE & Maclura tinctoria (L.) D.Don ex Steud. & Tajuva \\
\hline $\mathrm{C}$ & Rauh & MYRTACEAE & Eugenia uniflora L. & Pitanga \\
\hline $\mathrm{C}$ & Rauh & RUTACEAE & Helietta apiculata Benth. & Canela-de-veado \\
\hline $\mathrm{C}$ & Roux & LAURACEAE & Nectandra megapotamica (Spreng.) Mez & Canela-merda \\
\hline $\mathrm{C}$ & Roux & PRIMULACEAE & Myrsine loefgrenii (Mez) Imkhan. & Capororoca \\
\hline $\mathrm{C}$ & Roux & SALICACEAE & Casearia decandra Jacq. & $\begin{array}{l}\text { Guaçatonga- } \\
\text { branca }\end{array}$ \\
\hline $\mathrm{C}$ & Roux & SALICACEAE & Casearia sylvestris $\mathrm{Sw}$. & Guaçatonga-preta \\
\hline $\mathrm{C}$ & Scarrone & MELIACEAE & Cabralea canjerana (Vell.) Mart. & Canjarana \\
\hline $\mathrm{C}$ & Scarrone & MELIACEAE & Cedrela fissilis Vell. & Cedro-branco \\
\hline
\end{tabular}


TABELA 2: Continuação...

TABLE 2: Continued..

\begin{tabular}{|c|c|c|c|c|}
\hline P.R. & M.A. & Família & Nome científico & Nome vernacular \\
\hline $\mathrm{C}$ & Scarrone & RUBIACEAE & Randia ferox (Cham. \& Schltdl.) DC. & $\begin{array}{l}\text { Limoeiro-do- } \\
\text { mato }\end{array}$ \\
\hline $\mathrm{C}$ & Scarrone & RUTACEAE & Pilocarpus pennatifolius Lem. & Jaborandí \\
\hline $\mathrm{C}$ & Scarrone & RUTACEAE & Zanthoxylum rhoifolium Lam. & $\begin{array}{l}\text { Mamica-de- } \\
\text { cadela }\end{array}$ \\
\hline $\mathrm{D}$ & Champagnat & SAPINDACEAE & $\begin{array}{l}\text { Allophylus edulis (A.St.-Hil. et al.) Hieron. ex } \\
\text { Niederl. }\end{array}$ & Chal-chal \\
\hline $\mathrm{D}$ & Champagnat & SAPINDACEAE & Cupania vernalis Cambess. & Camboatá \\
\hline $\mathrm{D}$ & Champagnat & SAPINDACEAE & Matayba elaeagnoides Radlk. & Camboatá-branco \\
\hline $\mathrm{D}$ & Troll & ANNONACEAE & Annona neosalicifolia H.Rainer & Araticum \\
\hline $\mathrm{D}$ & Troll & FABACEAE & Albizia niopoides (Spruce ex Benth.) Burkart & Angico-branco \\
\hline $\mathrm{D}$ & Troll & FABACEAE & Enterolobium contortisiliquum (Vell.) Morong & Timbaúva \\
\hline $\mathrm{D}$ & Troll & FABACEAE & Inga vera Willd. & Ingá-banana \\
\hline $\mathrm{D}$ & Troll & FABACEAE & Parapiptadenia rigida (Benth.) Brenan & Angico-vermelho \\
\hline $\mathrm{D}$ & Troll & MALVACEAE & Luehea divaricata Mart. \& Zucc. & Açoita-cavalo \\
\hline $\mathrm{D}$ & Troll & MYRTACEAE & Campomanesia xanthocarpa $\mathrm{O}$. Berg & Guabiroba \\
\hline $\mathrm{D}$ & Troll & MYRTACEAE & Plinia rivularis (Cambess.) Rotman & Guapuriti \\
\hline $\mathrm{D}$ & Troll & POLYGONACEAE & Ruprechtia laxiflora Meisn. & $\begin{array}{l}\text { Marmeleiro-do- } \\
\text { mato }\end{array}$ \\
\hline $\mathrm{D}$ & Troll & SALICACEAE & Banara tomentosa $\mathrm{Clos}$ & Cambroé \\
\hline $\mathrm{D}$ & Troll & SAPOTACEAE & $\begin{array}{c}\text { Chrysophyllum gonocarpum (Mart. \& Eichler ex } \\
\text { Miq.) Engl. }\end{array}$ & Aguaí-da-serra \\
\hline $\mathrm{D}$ & Troll & SAPOTACEAE & Chrysophyllum marginatum (Hook. \&Arn.) Radlk. & Aguaí-vermelho \\
\hline
\end{tabular}

As espécies Casearia decandra, Casearia sylvestris, Myrsine loefgrenii e Nectandra megapotamica foram classificadas no modelo de Roux. As espécies Cabralea canjerana, Cedrela fissilis, Pilocarpus pennatifolius, Randia ferox e Zanthoxylum rhoifolium foram classificadas no modelo de Scarrone. As espécies Boehmeria caudata, Solanum sanctaecatharinae e Urera baccifera foram classificadas no modelo de Aubreville. No modelo de Attim, apenas foi classificada a espécie Moquiniastrum polymorphum.

O único modelo sem ramificação encontrado na pesquisa foi para uma palmeira, que não possui crescimento secundário mas tem hábito/porte arbóreo, Syagrus romanzoffiana, classificada como Modelo de Corner.

Nos modelos mais complexos, com eixos mistos, foram classificadas três espécies no modelo de Champagnat, entre elas: Allophylus edulis, Cupania vernalis, Matayba elaeagnoides. O modelo de Troll é um dos mais comuns no remanescente, 12 espécies foram classificadas pelo modelo de Troll, foram elas: Albizia niopoides, Annona neosalicifolia, Banara tomentosa, Campomanesia xanthocarpa, Chrysophyllum gonocarpum, Chrysophyllum marginatum, Enterolobium contortisiliquum, Inga vera, Luehea divaricata, Parapiptadenia rigida, Plinia rivularis e Ruprechtia laxiflora.

A chave dicotômica (Tabela 3) auxilia a identificação dos modelos classificados para as espécies da área amostrada. Para facilitar o entendimento, foram colocados exemplos de algumas espécies. 
TABELA 3: Chave dicotômica dos 23 modelos arquiteturais, preparada a partir de caracteres da arquitetura de árvores. Os modelos encontrados no presente trabalho são apresentados em negrito e as espécies são citadas como exemplo (Ex.).

TABLE 3: Key to the Architectural Models of Trees, prepared by elements of tree architecture. The models founded in the survey are presented with emphasis and the sampled species are cited like example (Ex.).

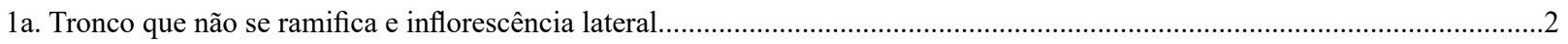

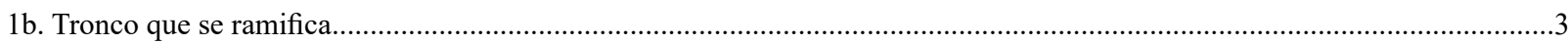

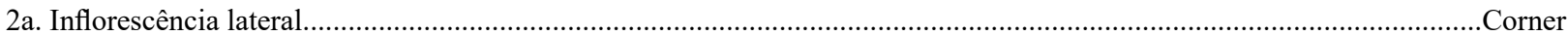

Ex. Syagrus romanzoffiana

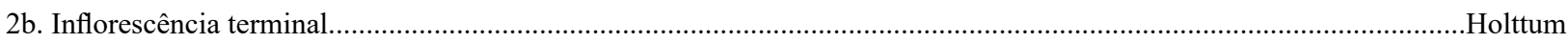

3a. Eixos vegetativos equivalentes, na maioria das vezes ortotrópicos e modulares..............................................................

3b. Eixos vegetativos não equivalentes (diferença entre troco e ramo) …...................................................................................

4a. Ramos na base do tronco, geralmente subterrâneos, normalmente, um crescimento contínuo.....................................Tomlinson

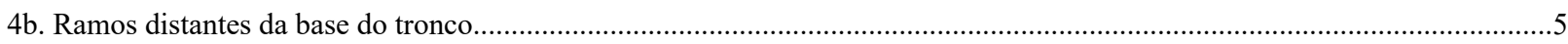

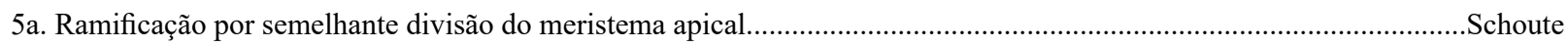

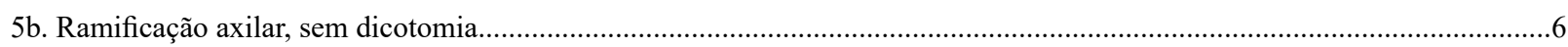

6a. Apenas um ramo por módulo, simpodial, linear e aparentemente não ramificado................................................Chamberlain

6b. Dois ou mais ramos por módulo; simpodial, com ramificação bem definida; inflorescência terminal.......................eewenberg

Ex. Pisonia zapallo; Schefflera morototoni.

7a. Eixos vegetativos heterogêneos, diferenciados em ortotrópicos e plagiotrópicos ou complexos..............................................8

7b. Eixos vegetativos homogêneos, ou todos ramos ortotrópicos ou mistos.............................................................................18

8a. Ramos na base do tronco, geralmente subterrâneos, normalmente um crescimento contínuo..............................................Clure

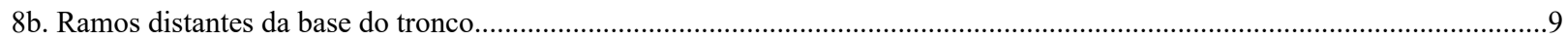

9a. Construção modular, ramos plagiotrópicos, normalmente com inflorescências terminais ou sésseis.........................................10

9b. Construção não modular, inflorescências normalmente laterais.........................................................................................13

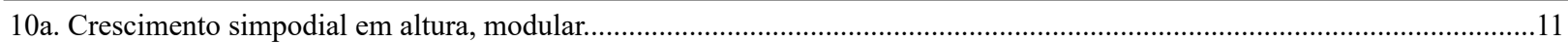

10b. Crescimento monopodial em altura, construção modular restrita aos ramos...............................................................12

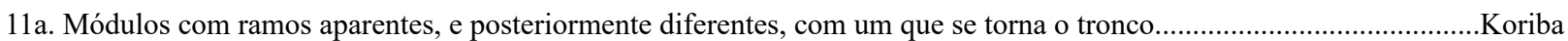

Ex. Handroanthus heptaphyllus; Phytolacca dioica

11b. Módulos diferenciados, com tronco surgindo posteriormente ao ramo, sendo bem diferenciados.................................Prevost

Ex. Cordia americana; Cordia trichotoma

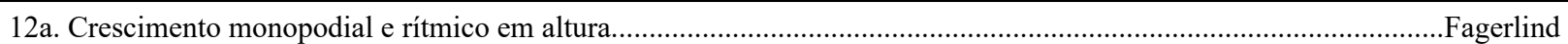

12b. Crescimento monopodial e contínuo em altura........................................................................................................

13a. Tronco com um crescimento simpodial de eixos ortotrópicos....................................................................................................

\section{Ex. Citronella paniculata}

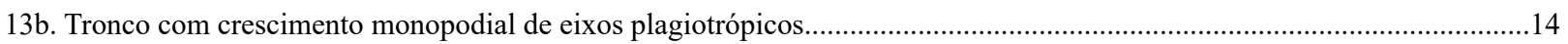

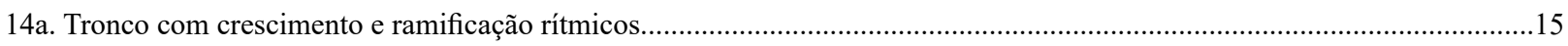

14b. Tronco com crescimento e ramificação contínuos ou difusos.......................................................................................16

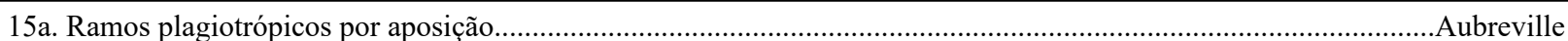

Ex. Boehmeria caudata; Solanum sanctaecatharinae; Urera baccifera

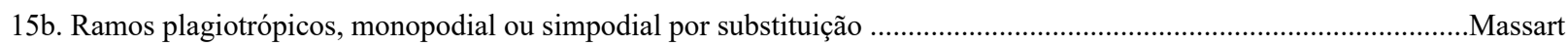

Ex. Diospyros inconstans; Eugenia involucrata; Eugenia ramboi; Myrcianthes pungens

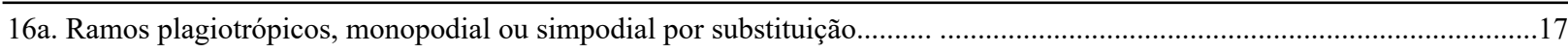

16b. Ramos plagiotrópicos por aposição............................................................................................................... Teórico 
TABELA 3: Continuação...

TABLE 3: Continued..

17a. Ramos longevos, sem semelhança com uma folha composta. Roux

Ex. Casearia decandra; Casearia sylvestris; Myrsine loefgrenii, Nectandra megapotamica

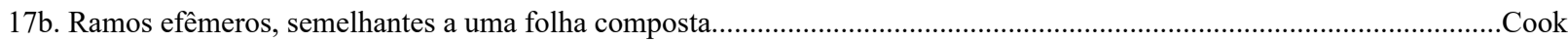

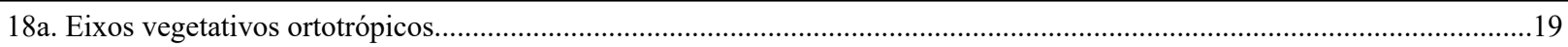

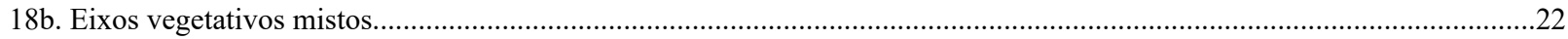

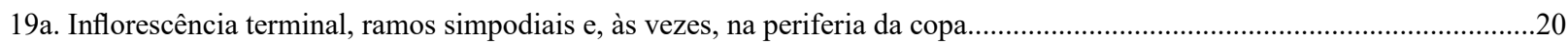

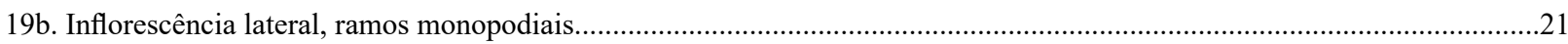

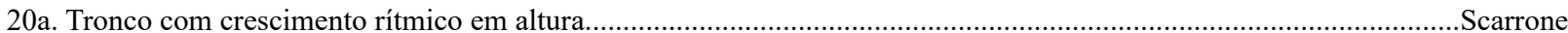

Ex. Cabralea canjerana; Cedrela fissilis; Pilocarpus pennatifolius; Randia ferox; Zanthoxylum rhoifolium.

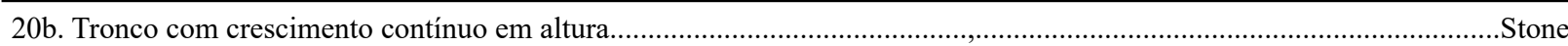

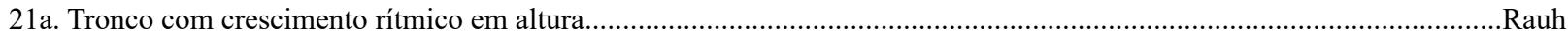

Ex. Actinostemon concolor; Aiouea saligna; Erythroxylum deciduum; Eugenia uniflora; Ficus luschnathiana; Helietta apiculata; Maclura tinctoria; Sebastiania brasiliensis; Sebastiania commersoniana; Trichilia catigua; Trichilia claussenii; Trichilia elegans; Vitex megapotamica

21b. Tronco com crescimento contínuo em altura Attim

Ex. Gochnatia polymorpha

19a. Inflorescência terminal, ramos simpodiais, às vezes, na periferia da copa.....................................................................20

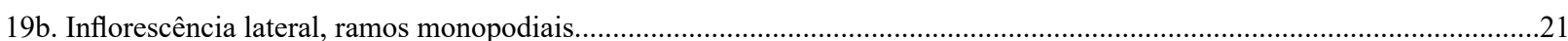

22a. Eixos mistos no crescimento primário, ramos basais ortotrópicos e distais plagiotrópicos..........................................angenot

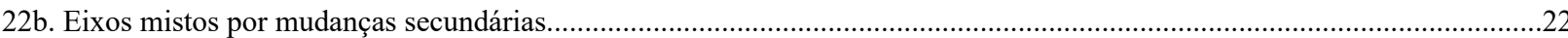

23a.Todos eixos ortotrópicos, posteriormente flertados

Champagnat

Ex. Allophylus edulis, Cupania vernalis, Matayba elaeagnoides

23b. Todos eixos plagiotrópicos, posteriormente eretos, após a queda das folhas. Troll

Ex. Albizia niopoides; Annona neosalicifolia; Banara tomentosa; Campomanesia xanthocarpa; Chrysophyllum gonocarpum; Chrysophyllum marginatum; Enterolobium contortisiliquum; Inga vera; Luehea divaricata; Parapiptadenia rígida; Plinia rivularis e Ruprechtia laxiflora.

Dos 23 modelos descritos por Hallé, Oldeman e Tomlinson (1978) foram registrados para a Floresta Estacional Subtropical Decidual estudada, 53 espécies distribuídas em 13 modelos arquitetônicos. Em uma Floresta Estacional Tropical Semidecidual da Península de Yucatán, no México, Vester (2002) registrou 108 espécies distribuídas em 15 modelos arquitetônicos. Em estudo sobre a arquitetura de uma Floresta Pluvial Tropical Perenifólia, na Amazônia Colombiana, 306 espécies distribuídas em 17 modelos arquitetônicos (VESTER; SALDARRIAGA, 1993). Estes números não tão distantes de modelos entre as florestas pluviais mencionadas e a florestas estacional subtropical corrobora as expectativas de Hallé, Oldeman e Tomlinson (1978) quando mencionam que o número de modelos decresce com o aumento do estresse ambiental advindo da sazonalidade, mais presente em florestas estacionais do que em florestas pluviais.

Os modelos mais encontrados na Floresta estudada foram o Rauh (13) e Troll (12), assim como estes modelos são abundantes nas regiões tropicais e em zonas temperadas e seu sucesso na natureza ocorre, de acordo com Hallé, Oldeman e Tomlinson (1978), devido à flexibilidade na construção de sua estrutura arbórea (VESTER, 2002). Na região neotropical, o modelo de Prevost está representado principalmente por espécies do gênero Cordia (HALLÉ; OLDEMAN; TOMLINSON, 1978, VESTER, 1997; 2002), também encontrado em duas espécies neste estudo.

A ausência de outros modelos pode ser explicada, na maioria dos casos, como modelos típicos de arbustos ou ervas. O modelo de Stone é muito raro na natureza e é encontrado principalmente no gênero Pandanus (Madagáscar) e algumas ervas, principalmente da família Asteraceae (HALLÉ; OLDEMAN; TOMLINSON, 1978). Outro modelo escasso é o de Schoute, que é limitado às monocotiledôneas e algumas espécies da família Cactaceae, encontrada no México (HALLÉ; OLDEMAN; TOMLINSON, 1978). O 
modelo Holttum não foi registrado, no entanto, é encontrado em espécies da família Bromeliaceae, que crescem naturalmente no solo (Gravatá - Bromelia balansae Meze). O modelo Tomlinson é escasso entre árvores, de modo que não foi registrado no trabalho. O modelo McClure também não é característico entre as árvores. As plantas encontradas no fragmento que são deste modelo são as taquaras da família Poaceae (Merostachys sp). Estes modelos não foram amostrados porque as espécies não possuem hábito arbóreo.

\section{CONCLUSÃO E RECOMENDAÇÕES}

Os modelos arquitetônicos mais encontrados para uma Floresta Estacional Subtropical foram os de Rauh, Troll, Scarrone, Roux e Massart; A complexidade arquitetural, refletida pelo elevado número de modelos descritos, se deve ao desenvolvimento do dossel no remanescente estudado; A riqueza de modelos arquitetônicos de espécies arbóreas em Florestas Estacionais é menor que da Floresta Pluvial; Este trabalho teve um caráter pioneiro e exploratório e deve fornecer um marco para novos estudos sobre arquitetura de plantas na região subtropical do Brasil.

\section{REFERÊNCIAS}

BARTHÉLÉMY, D.; CARAGLIO, Y. Plant architecture: a dynamic, multilevel and comprehensive approach to plant form, structure and ontogeny. Annals of Botany, London, v. 99, n. 3, p. 375-407, 2007.

HALLÉ, F. Arquitectura de los árboles. Boletin de la Sociedad Argentina de Botánica, Córdoba, v. 45, n. 3/4, p. 405-418, 2009.

HALlÉ, F.; OLDEMAN, R. A. A.; TOMLINSON, P. B. Tropical Trees and forests: an architectural analysis. Berlin: Springer-Verlag, 1978. 441 p.

LEITE, P. F.; KLEIN, R. M. Vegetação. In: IBGE. Geografia do Brasil: Região Sul. Rio de Janeiro: IBGE, 1990. p. 113-150.

OLDEMAN, R. A. A. Forests: elements of silvology. Berlin: Springer-Verlag, 1990. 624 p.

PERRETA, M. G.; VEGETTI, A. C. Patrones estructurales em las plantas vasculares: una revision. Gayana Botanica, Concepcion, v. 62, n. 1, p. 9-19, 2005.

TORQUEBIAU, E. F. Mosaic patterns in dipterocarps forest in Indonesia, and their implications for practical forestry. Journal of Tropical Ecology, Cambridge, v. 2, p. 301-325, 1986.

TORQUEBIAU, E. F. The reiteration of the architectural model, a demographic approach to the tree. 1979. 55 f. Tesis (Maestría) - Academie de Montpellier, Montpellier, 1979.

TOURN, G. M.; BARTHÉLÉMY, D.; GROSFELD, J. Una aproximación a la arquitectura: conceptos, objetivos y metodologia. Boletin de la Sociedad Argentina de Botánica, Córdoba, v. 32, n. 1-2, p. 85-99, 1999.

VESTER, H. F. M. Architectural diversification within the genus Vismia (Clusiaceae) in Amazonian rain forest (Araracuara, Colombia). In: KURMANN, M. H.; HEMSLEY, A. R. (Ed.). The evolution of plant architecture. Kew: Royal Botanic Gardens, 1999. p. 147-158.

VESTER, H. F. M. Modelos arquitectônicos em La Flora arbórea de La península de Yucatán. Boletín de la Sociedad Botánica de México, Distrito Federal, n. 71, p. 45-57, 2002.

VESTER, H. F. M. The trees and the forest. The role of tree architecture in canopy development; a case study in secondary forests (Araracuara, Colombia). 1997. 180 f. Tesis (Doctoral) Universiteit van Amsterdam, Amsterdam, 1997.

VESTER, H. F. M.; CLEEF, A. M. Tree architecture and secondary tropical rain forest development: a case study in Araracuara, Colombian Amazonia. Flora, [S.1.] n. 193, p. 75-97, 1998.

VESTER, H. F. M.; SALDARRIAGA, J. Algunas características estructurales, arquitectónicas y florísticas de la sucesión secundaria sobre Terrazas Bajas em la región de Araracuara (Colombia). Revista de La Facultad Nacional de Agronomía, Medellín, v. 1, n. 2, p. 15-45, 1993. 